\title{
Access to Original Vinylic Chlorides in the Quinazoline Series via a Monoelectronic Transfer Reaction Approach
}

\author{
Martine Maillard-Boyer, Caroline Castera-Ducros, Pierre Verhaeghe, France Sifredi, \\ Pascal Rathelot and Patrice Vanelle*
}

Laboratoire de Pharmacochimie Radicalaire, Faculté de Pharmacie, Universités d'Aix-Marseille I, II et III - UMR CNRS 6264, Laboratoire Chimie Provence, 27 Boulevard Jean Moulin, 13385 Marseille cedex 05, France; E-Mails: martine.maillard@univmed.fr (M.M.); caroline.ducros@univmed.fr (C.C.); pierre.verhaeghe@univmed.fr(P.V.); france.sifredi@univmed.fr (F.S.); pascal.rathelot@univmed.fr (P.R.)

* Author to whom correspondence should be addressed; E-mail: patrice.vanelle@univmed.fr; Tel.: +33 491835 528, Fax: +33 491794677 .

Received: 26 January 2010; in revised form: 21 February 2010 / Accepted: 12 April 2010 / Published: 13 April 2010

Abstract: A series of new quinazoline derivatives bearing a vinylic chloride group on the 2-position was prepared by using a consecutive $\mathrm{S}_{\mathrm{RN}} 1 / \mathrm{E}_{\mathrm{RC}} 1$ radical strategy.

Keywords: $\mathrm{S}_{\mathrm{RN}} 1$; $\mathrm{E}_{\mathrm{RC}} 1$; quinazoline ring; vinylic chlorides

\section{Introduction}

The quinazoline ring is of major pharmaceutical interest, considering both that it is the molecular scaffold of several drug-compounds (Erlotinib, Lapatinib, Prazozin, Alfuzosin...) and, that 4-anilinosubstituted-quinazolines are key structures for the development of new selective anticancer chemotherapies, as kinase inhibitors. Focusing on the synthesis of new 2,4-bisubstituted-quinazolines, with a scope of studying their biological properties, our research team also discovered that some 4-anilinoquinazolines have original in vitro antiplasmodial properties [1-2]. Quite recently, we prepared some 4-anilinoquinazolines, substituted at position 6 with a nitro group and at position 2 with a vinylic group, one of them exerting in vitro antiplasmodial properties [3]. To explore more precisely the relations between chemical structure and biological activity, we studied the possibility of synthesizing new 4-aminated-quinazoline derivatives presenting a vinylic chloride substituent at 
position 2. In order to access to such vinylic chloride group, different strategies were developed among which the one presented by Ryabukhin et al. [4] and the use of trichloromethylated heterocycles, as substrates for the radical $\mathrm{S}_{\mathrm{RN}} 1+\mathrm{E}_{\mathrm{RC}} 1$ reaction with nitroalkanes, successful approach in isoquinoline, imidazole and quinoline series [5-7]. We describe herein, the use of the consecutive $\mathrm{S}_{\mathrm{RN}} 1+\mathrm{E}_{\mathrm{RC}} 1$ reaction from 2-trichloromethylquinazoline substrates and nitroalkanes, for the preparation of these original vinylic chloride derivatives in a rapid and convenient way.

\section{Results and Discussion}

Since 2009, it is known that 2-chloromethyl-6-nitroquinazoline derivatives can react with nitroalkanes through a $\mathrm{S}_{\mathrm{RN}} 1$ mechanism [8]. However, the $\mathrm{S}_{\mathrm{RN}} 1$ reaction involving a radical anion intermediate which is generally stabilized with the presence of a nitro group properly located on the heterocycle, it was not obvious that an aminated quinazoline substrate would react, because of the electrodonating effect of this group.

First, the key-precursor 1 was prepared from commercial 2-methylquinazolin-4(3H)-one via a microwave-assisted chlorination procedure using phosphorus pentachloride in phosphorus oxychloride [9]. Then, after proceeding to a solvent free $\mathrm{S}_{N} A r$ reaction with $n$-propyl- and $n$ butylamines, the corresponding intermediates $\mathbf{2}$ and $\mathbf{3}$ were nitrated in classical conditions to afford $\mathbf{4}$ and 5 in good yields (Scheme 1).

Scheme 1. Two step preparation of the radical reaction substrates $\mathbf{4}$ and $\mathbf{5}$.<smiles>Clc1nc(C(Cl)(Cl)Cl)nc2ccccc12</smiles>

1 equiv.

1<smiles>[R12]Nc1nc(C(C)(Cl)Cl)nc2ccccc12</smiles>

2: $\mathrm{R}_{1}=\mathrm{C}_{3} \mathrm{H}_{7} 95 \%$

3: $\mathrm{R}_{1}=\mathrm{C}_{4} \mathrm{H}_{9} 73 \%$

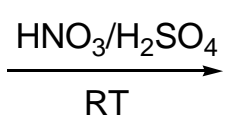<smiles>[R1]Nc1nc(C(Cl)(Cl)Cl)nc2ccc([N+](=O)[O-])cc12</smiles>

$4: \mathrm{R}_{1}=\mathrm{C}_{3} \mathrm{H}_{7} 85 \%$

$5: \mathrm{R}_{1}=\mathrm{C}_{4} \mathrm{H}_{9} 75 \%$

Then, compound 4, which is prepared in higher global yield, was chosen as a substrate for studying the SRN1+ERC1 reaction with 2-nitropropane. The first reaction assay, at room temperature (RT) under N2 atmosphere and light irradiation, enabled us to isolate both vinylic chloride 6 and the dimer of 2-nitropropane 7 (Scheme 2).

Scheme 2. General presentation of the $\mathrm{S}_{\mathrm{RN}} 1+\mathrm{E}_{\mathrm{RC}} 1$ reaction of substrate 4 with 2-nitropropane.<smiles>CCCNc1nc(C(Cl)(Cl)Cl)nc2ccc([N+](=O)[O-])cc12</smiles>

4<smiles>CCCNc1nc(C(Cl)=C(C)C)nc2ccc([N+](=O)[O-])cc12</smiles>

6

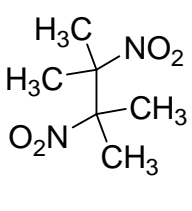

7 
In order to optimize the reaction, several experimental conditions were studied, especially the ones described by Kornblum et al. [10] (lithium salt of 2-nitropropane in DMSO or DMF) and Norris et al. [11] (phase transfer conditions using $\mathrm{TBAOH}$ and 2-nitropropane). The results showed that the phase transfer conditions of Norris gave the best reaction yield. The amount of 2-nitropropane required was also studied, indicating that the reaction yield is globally optimal (82\%) when using 5 equiv. (Table 1 , entries 1-6). Reaction progress was monitored by TLC, and appeared to be complete after 4 hours.

The formation of dimer 7 clearly suggested a consecutive $\mathrm{S}_{\mathrm{RN}} 1+\mathrm{E}_{\mathrm{RC}} 1$ reaction mechanism. To validate this hypothesis, we studied the influence of several radical reaction inhibitors (Table 1 entries 7-10). The results showed that the reaction obeys to a radical pathway, in particular when comparing run 3 with runs 8 or 9 . The synthesis of vinylic chloride 6 in low yield, when using 1 equiv. of $p$-dinitrobenzene (assay 10), may be due to the insufficient difference in reduction potential between $p$-dinitrobenzene and substrate 4 , partially permitting the monoelectronic reduction of this last by the 2-nitropropane anion, followed by the formation of product $\mathbf{6}$, through complete $\mathrm{S}_{\mathrm{RN}} 1+\mathrm{E}_{\mathrm{RC}} 1$ reaction sequence.

Table 1. Reactivity of substrate 4 with 2-nitropropane.

\begin{tabular}{cccccc}
\hline Run* & $\mathbf{M}^{+}$ & Solvent & $\begin{array}{c}\text { 2-Nitropropane } \\
\text { (equiv.) }\end{array}$ & Inhibitor & Yield of 6 (\%) \\
\hline $\mathbf{1}$ & $\mathrm{NBu}_{4}$ & $\mathrm{CH}_{2} \mathrm{Cl}_{2} / \mathrm{H}_{2} \mathrm{O}$ & 2 & - & 60 \\
$\mathbf{2}$ & $\mathrm{NBu}_{4}$ & $\mathrm{CH}_{2} \mathrm{Cl}_{2} / \mathrm{H}_{2} \mathrm{O}$ & 4 & - & 75 \\
$\mathbf{3}$ & $\mathrm{NBu}_{4}$ & $\mathrm{CH}_{2} \mathrm{Cl}_{2} / \mathrm{H}_{2} \mathrm{O}$ & 5 & - & 82 \\
$\mathbf{4}$ & $\mathrm{NBu}_{4}$ & $\mathrm{CH}_{2} \mathrm{Cl}_{2} / \mathrm{H}_{2} \mathrm{O}$ & 6 & - & 83 \\
$\mathbf{5}$ & $\mathrm{Li}$ & $\mathrm{DMF}$ & 5 & - & 53 \\
$\mathbf{6}$ & $\mathrm{Li}$ & $\mathrm{DMSO}$ & 5 & - & 70 \\
$\mathbf{7}$ & $\mathrm{Li}$ & $\mathrm{DMSO}$ & 5 & $\mathrm{CuCl}_{2}(1$ equiv.) & 25 \\
$\mathbf{8}$ & $\mathrm{NBu}_{4}$ & $\mathrm{CH}_{2} \mathrm{Cl}_{2} / \mathrm{H}_{2} \mathrm{O}$ & 5 & $\mathrm{TEMPO}_{1}(1$ equiv.) & 0 \\
$\mathbf{9}$ & $\mathrm{NBu}_{4}$ & $\mathrm{CH}_{2} \mathrm{Cl}_{2} / \mathrm{H}_{2} \mathrm{O}$ & 5 & $\mathrm{O}_{2}$ bubbling & 0 \\
$\mathbf{1 0}$ & $\mathrm{NBu}_{4}$ & $\mathrm{CH}_{2} \mathrm{Cl}_{2} / \mathrm{H}_{2} \mathrm{O}$ & 5 & $p$-dinitrobenzene & 35 \\
\hline
\end{tabular}

*Reaction conditions: $\mathrm{N}_{2}$ atmosphere, $60 \mathrm{~W}$ white light irradiation, RT. Reactions were monitored by TLC.

From the optimal reaction conditions previously presented, we then extended the reaction to other nitroalkanes, in order to prepare several vinylic chlorides in quinazoline series (Scheme 3). So the same radical reaction was conducted with two other secondary nitroalkanes (nitrocycloalkanes) and three primary nitroalkanes. In the reactions using secondary nitroalkanes, the corresponding vinylic chlorides were obtained in identical yields $(60-82 \%)$ as with nitroalkane dimers, whereas in the reactions involving primary nitroalkanes, only the corresponding vinylic chlorides were obtained, in significantly lower yields $(50-56 \%)$, as presented in Table 2. Concerning the vinylic chlorides prepared from primary nitroalkanes, the analysis of 2D Noesy NMR spectra revealed a $Z$ configuration for the vinylic double bond.

Such results appeared identical to the ones obtained with the $\mathrm{S}_{\mathrm{RN}} 1+\mathrm{E}_{\mathrm{RC}} 1$ reaction operated in 2trichloromethylquinoline series [7], but demonstrate that this reaction can be compatible with the presence of an electrodonating group on the heterocyclic scaffold. 
Scheme 3. Optimal reaction conditions for the synthesis of vinylic chloride derivatives from 4.<smiles>CCCNc1nc(C(Cl)(Cl)Cl)nc2ccc([N+](=O)[O-])cc12</smiles>

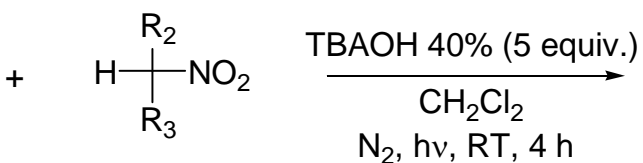

5 equiv.
$\mathrm{N}_{2}, \mathrm{~h} v, \mathrm{RT}, 4 \mathrm{~h}$<smiles>[R]C([R])=C(Cl)c1nc(NCCC)c2cc([N+](=O)[O-])ccc2n1</smiles>

$50-82 \%$

Table 2. Series of vinylic chloride derivatives prepared from 4, using optimal reaction conditions.

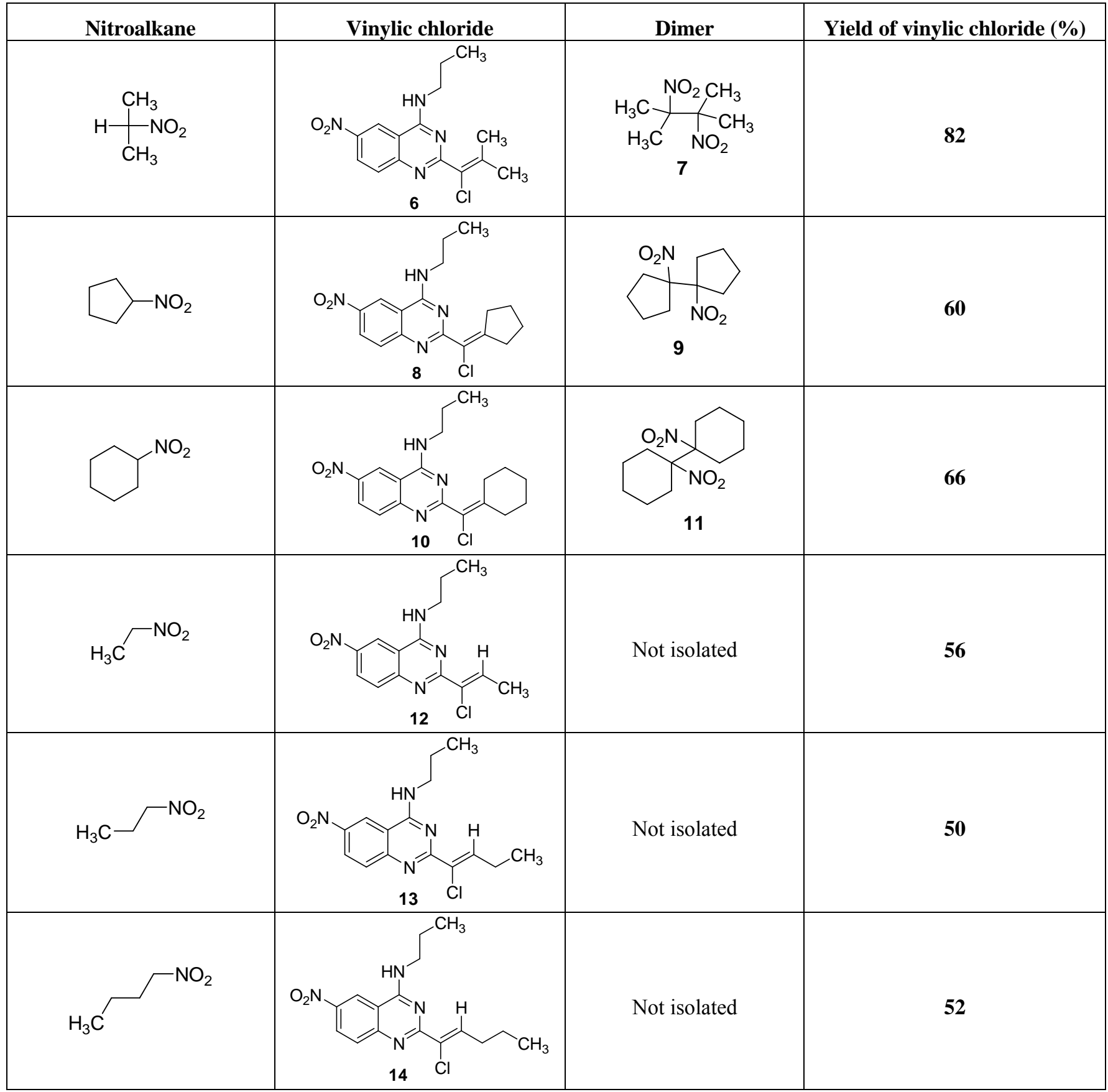


To enlarge the reaction spectrum, we involved two other aminated substrates in the same reaction with 2-nitropropane, using the previously optimal reaction conditions. Substrate 5, containing an other primary amine substituent, was prepared in parallel with $\mathbf{4}$ and afforded vinylic chloride $\mathbf{1 5}$ in $57 \%$ yield (Scheme 4).

Scheme 4. Extension of the reaction with substrate 5.<smiles>CCCCNc1nc(C(Cl)(Cl)Cl)nc2ccc([N+](=O)[O-])cc12</smiles>

5<smiles>CC(C)[N+](=O)[O-]</smiles>

5 equiv.<smiles>CCCCNc1nc(C(Cl)=C(C)C)nc2ccc([N+](=O)[O-])cc12</smiles>

$1557 \%$

Aiming at preparing a vinylic chloride product bearing an anilino substituent at position 4 of quinazoline, we had to prepare an appropriate substrate candidate for the $S_{R N} 1+E_{R C} 1$ reaction. Thus, since the nitration reaction could not be done after the introduction of the aniline onto the quinazoline ring, the synthetic strategy from 2-methylquinazolin-4(3H)-one was modified, starting by a nitration step, under classical conditions. After successive chlorination and solvent free $\mathrm{S}_{\mathrm{N}} \mathrm{Ar}$ reactions, 18 was obtained (Scheme 5).

Scheme 5. Three step preparation of substrate 18.<smiles>Cc1nc2ccccc2c(=O)[nH]1</smiles>

So we applied the optimal reaction conditions defined previously to the reaction of $\mathbf{1 8}$ with 2-nitropropane and obtained the expected vinylic chloride 19 in 45\% yield (Scheme 6).

Scheme 6. $\mathrm{S}_{\mathrm{RN}} 1+\mathrm{E}_{\mathrm{RC}} 1$ reaction of 18 with 2-nitropropane.<smiles>O=[N+]([O-])c1ccc2nc(C(F)(F)F)nc(Nc3cccc(C(F)(F)F)c3)c2c1</smiles>

1 equiv.

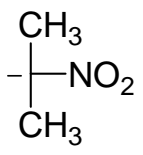
5 equiv.
$\underset{\mathrm{CH}_{2} \mathrm{Cl}_{2}}{\stackrel{\text { TBAOH }}{\longrightarrow} \text { (5 equiv.) }}$ $\mathrm{N}_{2}, \mathrm{hv}, \mathrm{RT}, 4 \mathrm{~h}$<smiles>CC(C)=C(Cl)c1nc(Nc2cccc(C(F)(F)F)c2)c2cc([N+](=O)[O-])ccc2n1</smiles>

$1945 \%$ 
When 2-methylquinazolin-4(3H)-one is first chlorinated and then nitrated, compound 20 is formed in $72 \%$ global yield (Scheme 7).

Scheme 7. Two step preparation of substrate 20.<smiles>Cc1nc2ccccc2c(=O)[nH]1</smiles><smiles>O=S(=O)(O)O[Na]</smiles>

$175 \%$<smiles>O=c1[nH]c(C(Cl)(Cl)Cl)nc2ccc([N+](=O)[O-])cc12</smiles>

$2096 \%$

Finally, because of its trichloromethyl group, we thought that such nitrated lactam ring 20 could be an interesting substrate for the $\mathrm{S}_{\mathrm{RN}} 1+\mathrm{E}_{\mathrm{RC}} 1$ reaction. Unfortunately, its low solubility in dichloromethane did not allow the use of the optimal phase transfer reaction conditions that we already presented. So we reacted 20 with the lithium salt of 2-nitropropane in DMSO (Kornblum conditions [10]) during 2 hours and formed the vinylic chloride product $\mathbf{2 1}$ in $67 \%$ yield, in addition to the dimer of 2-nitropropane 7, in similar yield (Scheme 8).

Scheme 8. $\mathrm{S}_{\mathrm{RN}} 1+\mathrm{E}_{\mathrm{RC}} 1$ reaction of $\mathbf{2 0}$ with the lithium salt of 2-nitropropane.<smiles>O=c1[nH]c(C(Cl)(Cl)Cl)nc2ccc([N+](=O)[O-])cc12</smiles>

20<smiles>CC(C)([18F])[N+](=O)[O-]</smiles>

5 equiv.<smiles>CC(C)=C(Cl)c1nc2ccc([N+](=O)[O-])cc2c(=O)[nH]1</smiles>

$2167 \%$<smiles>CC(C)([N+](=O)[O-])C(C)(C)[N+](=O)[O-]</smiles>

7

\section{Experimental}

\subsection{General}

Melting points were determined with a B-540 Büchi melting point apparatus. $200 \mathrm{MHz}{ }^{1} \mathrm{H}-\mathrm{NMR}$ and $50 \mathrm{MHz}{ }^{13} \mathrm{C}$-NMR spectra were recorded on a Brüker ARX 200 spectrometer in $\mathrm{CDCl}_{3}$ or DMSO$\mathrm{d}_{6}$ at the Faculté de Pharmacie de Marseille. ${ }^{1} \mathrm{H}$ - and ${ }^{13} \mathrm{C}-\mathrm{NMR}$ chemical shifts $(\delta)$ are reported in ppm with respect to $\mathrm{CDCl}_{3} 7.26 \mathrm{ppm}\left({ }^{1} \mathrm{H}\right), 77.2 \mathrm{ppm}\left({ }^{13} \mathrm{C}\right)$ or DMSO-d 6 2.50 ppm $\left({ }^{1} \mathrm{H}\right), 39.5\left({ }^{13} \mathrm{C}\right) .2 \mathrm{D}$ NOESY NMR spectra and elemental analyses were carried out at the Spectropole, Faculté des Sciences et Techniques de Saint-Jérôme. Silica gel 60 (Merck, particular size 0.063-0.200 mm, 70-230 mesh ASTM) was used for column chromatography. TLC was performed on $5 \mathrm{~cm} \times 10 \mathrm{~cm}$ aluminium plates coated with silica gel 60F-254 (Merck) in appropriate solvent. The following substrates or subproducts were prepared and described previously: 4-chloro-2-trichloromethylquinazoline (1) [9,12], N-butyl-2-trichloromethylquinazolin-4-amine (3) [13], 2,3-dimethyl-2,3dinitrobutane (7) [14], 1-nitro-1-(1-nitrocyclopentyl)cyclopentane (9) [15], 1-nitro-1-(1-nitrocyclo- 
hexyl)cyclohexane (11) [16], 2-methyl-6-nitroquinazolin-4(3H)one (16) [17], 4-chloro-6-nitro-2trichloromethylquinazoline (17) [18], and 6-nitro-2-trichloromethylquinazolin-4(3H)-one (20) [18].

\subsection{Preparation of N-propyl-2-trichloromethylquinazolin-4-amine (2)}

Three equiv. of propylamine were added onto 1 equiv. of 4-chloro-2-trichloromethylquinazoline (1). The solvent-free reaction mixture was stirred at $30{ }^{\circ} \mathrm{C}$ for $1 \mathrm{~min}$. The mixture was poured into a solution of $\mathrm{NaOH}(5 \%)$ and extracted with dichloromethane. The organic layer was washed with water three times, dried over anhydrous $\mathrm{Na}_{2} \mathrm{SO}_{4}$, concentrated in vacuo and recrystalized from cyclohexane to afford 2 in 95\% yield.Beige solid; yield 95\%, mp $136{ }^{\circ} \mathrm{C} .{ }^{1} \mathrm{H}-\mathrm{NMR}\left(\mathrm{CDCl}_{3}\right) \delta 1.03(3 \mathrm{H}$, t, $J=7.3 \mathrm{~Hz}), 1.69-1.87(2 \mathrm{H}, \mathrm{m}), 3.66-3.76(2 \mathrm{H}, \mathrm{m}), 6.06(1 \mathrm{H}$, br s$), 7.49-7.57(1 \mathrm{H}, \mathrm{m}), 7.73-7.81(2 \mathrm{H}$, m), $7.97(1 \mathrm{H}, \mathrm{d}, J=8.3 \mathrm{~Hz}) .{ }^{13} \mathrm{C}-\mathrm{NMR}\left(50 \mathrm{MHz} ; \mathrm{CDCl}_{3}\right) \delta 11.6\left(\mathrm{CH}_{3}\right), 22.5\left(\mathrm{CH}_{2}\right), 43.4\left(\mathrm{CH}_{2}\right), 98.4$ (C), $113.8(\mathrm{C}), 121.2(\mathrm{CH}), 127.4(\mathrm{CH}), 129.0(\mathrm{CH}), 133.2(\mathrm{CH}), 149.1(\mathrm{C}), 160.7(\mathrm{C}), 161.5(\mathrm{C})$. Anal. calcd for $\mathrm{C}_{12} \mathrm{H}_{12} \mathrm{Cl}_{3} \mathrm{~N}_{3}$ : C, 47.32; H, 3.97; N, 13.80. Found: C, 47.90; H, 4.24; N, 13.85 .

\subsection{General Procedure for the Preparation of Compounds $\mathbf{4}$ and $\mathbf{5}$}

To concentrated sulfuric acid ( $25 \mathrm{~mL}), 1$ equiv. of $N$-alkyl-2-trichloromethylquinazolin-4-amine (2) or 3 was added at $0{ }^{\circ} \mathrm{C}$. Then, 5 equiv. of concentrated nitric acid were added slowly. The reaction mixture was stirred for $5 \mathrm{~h}$ at $\mathrm{rt}$. The reaction mixture was then slowly poured into a water-ice mixture and alkalinized with sodium carbonate, making the desired products precipitate. The suspension was filtered and the precipitate was dried under reduced pressure. After purification by chromatography on a silica gel column, eluting with dichloromethane, products 4 or 5 were recrystallized from ethyl acetate and obtained, respectively, in 85 and $75 \%$ yields.

6-Nitro-N-propyl-2-trichloromethylquinazolin-4-amine (4): Yellow solid; yield $85 \%, \mathrm{mp} 151{ }^{\circ} \mathrm{C} .{ }^{1} \mathrm{H}$ $\operatorname{NMR}\left(\mathrm{CDCl}_{3}\right) \delta 1.07(3 \mathrm{H}, \mathrm{t}, J=7.3 \mathrm{~Hz}), 1.76-1.94(2 \mathrm{H}, \mathrm{m}), 3.74-3.84(2 \mathrm{H}, \mathrm{m}), 6.58(1 \mathrm{H}, \mathrm{br} \mathrm{s}), 8.07(1 \mathrm{H}$, $\mathrm{d}, J=9.1 \mathrm{~Hz}), 8.54(1 \mathrm{H}, \mathrm{dd}, J=9.1 \mathrm{~Hz}$ and $J=2.4 \mathrm{~Hz}), 8.83(1 \mathrm{H}, \mathrm{d}, J=2.4 \mathrm{~Hz}) .{ }^{13} \mathrm{C}-\mathrm{NMR}\left(\mathrm{CDCl}_{3}\right) \delta$ $11.7\left(\mathrm{CH}_{3}\right), 22,5\left(\mathrm{CH}_{2}\right), 44.2\left(\mathrm{CH}_{2}\right), 97.3(\mathrm{C}), 113.1(\mathrm{C}), 118.5(\mathrm{CH}), 127,2(\mathrm{CH}), 130.8(\mathrm{CH}), 145.7$ (C), 152.7 (C), 161.6 (C), 164.4 (C). Anal. Calcd for $\mathrm{C}_{12} \mathrm{H}_{11} \mathrm{Cl}_{3} \mathrm{~N}_{4} \mathrm{O}_{2}$ : C, 41.23; H, 3.17; N, 16.03. Found: C, 41.74; H, 3.05; N, 16.45 .

N-Butyl-6-nitro-2-trichloromethylquinazolin-4-amine (5): Yellow solid; yield $75 \%, \mathrm{mp} 168{ }^{\circ} \mathrm{C}$. ${ }^{1} \mathrm{H}$ $\operatorname{NMR}\left(\mathrm{CDCl}_{3}\right) \delta 1.01(3 \mathrm{H}, \mathrm{t}, J=7.3 \mathrm{~Hz}), 1.40-1.59(2 \mathrm{H}, \mathrm{m}), 1.75-1.87(2 \mathrm{H}, \mathrm{m}), 3.82(2 \mathrm{H}, \mathrm{q}, J=12.7$ $\mathrm{Hz}), 6.75(1 \mathrm{H}$, br s), $8.07(1 \mathrm{H}, \mathrm{d}, J=9.2 \mathrm{~Hz}), 8.55(1 \mathrm{H}, \mathrm{dd}, J=9.2$ and $2.3 \mathrm{~Hz}), 8.83(1 \mathrm{H}, \mathrm{d}, J=2.3$ $\mathrm{Hz}) .{ }^{13} \mathrm{C}-\mathrm{NMR}\left(\mathrm{CDCl}_{3}\right) \delta 13.8\left(\mathrm{CH}_{3}\right), 20.1\left(\mathrm{CH}_{2}\right), 31.1\left(\mathrm{CH}_{2}\right), 41.9\left(\mathrm{CH}_{2}\right), 97.3(\mathrm{C}), 112.9(\mathrm{C}), 118.0$ $(\mathrm{CH}), 126.9(\mathrm{CH}), 131.1(\mathrm{CH}), 145.5(\mathrm{C}), 153.0(\mathrm{C}), 161.3(\mathrm{C}), 164.5(\mathrm{C})$. Anal. Calcd for $\mathrm{C}_{13} \mathrm{H}_{13} \mathrm{Cl}_{3} \mathrm{~N}_{4} \mathrm{O}_{2}$ : C, 42.94; H, 3.60; N, 15.41. Found: C, 43.01; H, 3.63; N, 15.25 .

\subsection{General Procedure for the Synthesis of Vinylic Chlorides 6, 8, 10, 12, 13, 14, 15 and 19}

Five equiv. each of both nitroalkane and 40\% TBAOH were stirred at room temperature for $30 \mathrm{~min}$ under nitrogen atmosphere. Then, 1 equiv. of 4-substituted-6-nitro-2-trichloromethylquinazoline substrate 4, 5 or 18, solubilized in dichloromethane, was added. The mixture was rapidly stirred at $\mathrm{rt}$ 
under nitrogen atmosphere with white light irradiation $(60 \mathrm{~W})$ for $4 \mathrm{~h}$. After disappearance of substrate (monitored by TLC), the reaction was stopped with addition of water and the product was extracted with dichloromethane. The organic layer, washed with water several times, was finally dried over anhydrous sodium sulphate and evaporated. The product was purified by chromatography on a silica gel, eluting with appropriate solvent, to give the corresponding vinylic chloride products.

2-(1-Chloro-2-methylprop-1-enyl)-6-nitro-N-propylquinazolin-4-amine (6): The eluent was petroleum ether-ethyl acetate 50:50. Yellow solid; yield 82\%, mp $145{ }^{\circ} \mathrm{C} .{ }^{1} \mathrm{H}-\mathrm{NMR}\left(\mathrm{CDCl}_{3}\right) \delta 1.06(3 \mathrm{H}, \mathrm{t}$, $J=7.3 \mathrm{~Hz}), 1.73-1.86(2 \mathrm{H}, \mathrm{m}), 2.05(3 \mathrm{H}, \mathrm{s}), 2.09$ (3H, s), 3.66-3.76 (2H, m), 6.29 (1H, br s), 7.94 $(1 \mathrm{H}, \mathrm{d}, J=9.2 \mathrm{~Hz}), 8.48(1 \mathrm{H}, \mathrm{dd}, J=9.2 \mathrm{~Hz}$ and $2.4 \mathrm{~Hz}), 8.76(1 \mathrm{H}, \mathrm{d}, J=2.4 \mathrm{~Hz}) .{ }^{13} \mathrm{C}-\mathrm{NMR}\left(\mathrm{CDCl}_{3}\right)$ $\delta 11.7\left(\mathrm{CH}_{3}\right), 22.4\left(\mathrm{CH}_{3}\right), 22.7\left(\mathrm{CH}_{2}\right), 23.3\left(\mathrm{CH}_{3}\right), 43.7\left(\mathrm{CH}_{2}\right), 112.5(\mathrm{C}), 118.4(\mathrm{CH}), 124.5(\mathrm{C}), 126.6$ (CH), $130.1(\mathrm{CH}), 137.7$ (C), 144.7 (C), 153.4 (C), 160.7 (C), 164.2 (C). Anal. Calcd for $\mathrm{C}_{15} \mathrm{H}_{17} \mathrm{ClN}_{4} \mathrm{O}_{2}$ : C, 56.16; H, 5.34; N, 17.47. Found: C, 56.75; H, 5.69; N, 17.05.

2-[Chloro(cyclopentylidene)methyl]-6-nitro-N-propylquinazolin-4-amine (8): The eluent was dichloromethane-ethyl acetate 98:2. Orange solid; yield 60\%, mp $160{ }^{\circ} \mathrm{C} .{ }^{1} \mathrm{H}-\mathrm{NMR}\left(\mathrm{DMSO}-\mathrm{d}_{6}\right) \delta 0.95$ $(3 \mathrm{H}, \mathrm{t}, J=7.3 \mathrm{~Hz}), 1.59-1.80(6 \mathrm{H}, \mathrm{m}), 2.59-2.65(2 \mathrm{H}, \mathrm{m}), 2.92-2.98(2 \mathrm{H}, \mathrm{m}), 3.49-3.51(2 \mathrm{H}, \mathrm{m}), 7.76$ $(1 \mathrm{H}, \mathrm{d}, J=9.2 \mathrm{~Hz}), 8.42(1 \mathrm{H}, \mathrm{dd}, J=9.2 \mathrm{~Hz}$ and $2.3 \mathrm{~Hz}), 8.98-9.00(1 \mathrm{H}, \mathrm{m}), 9.31(1 \mathrm{H}, \mathrm{d}, J=2.3 \mathrm{~Hz})$. ${ }^{13} \mathrm{C}-\mathrm{NMR}\left(\mathrm{DMSO}-\mathrm{d}_{6}\right) \delta 11.7\left(\mathrm{CH}_{3}\right), 21.8\left(\mathrm{CH}_{2}\right), 25.1\left(\mathrm{CH}_{2}\right), 27.8\left(\mathrm{CH}_{2}\right), 35.2\left(\mathrm{CH}_{2}\right), 36.6\left(\mathrm{CH}_{2}\right), 43.0$ $\left(\mathrm{CH}_{2}\right), 112.3(\mathrm{C}), 120.6(\mathrm{CH}), 122.4(\mathrm{C}), 126.5(\mathrm{CH}), 129.2(\mathrm{CH}), 144.0(\mathrm{C}), 153.4(\mathrm{C}), 154.2(\mathrm{C})$, 160.2 (C), $161.9(\mathrm{C})$. HR MS (+ESI): $m / z 347.1269\left(\mathrm{M}+\mathrm{H}^{+}\right)$. Calcd for $\mathrm{C}_{17} \mathrm{H}_{19} \mathrm{ClN}_{4} \mathrm{O}_{2}: 346.1197$.

2-[Chloro(cyclohexylidene)methyl]-6-nitro-N-propylquinazolin-4-amine (10): The eluent was petroleum ether-ethyl acetate 50:50. Yellow solid; yield $66 \%, \mathrm{mp} 170{ }^{\circ} \mathrm{C} .{ }^{1} \mathrm{H}-\mathrm{NMR}\left(\mathrm{CDCl}_{3}\right) \delta 1.05$ $(3 \mathrm{H}, \mathrm{t}, J=7.3 \mathrm{~Hz}), 1.61-1.85(10 \mathrm{H}, \mathrm{m}), 2.57-2.62(2 \mathrm{H}, \mathrm{m}), 3.66-3.76(2 \mathrm{H}, \mathrm{m}), 6.37$ (1H, br s), 7.93 $(1 \mathrm{H}, \mathrm{d}, J=9.2 \mathrm{~Hz}), 8.46(1 \mathrm{H}, \mathrm{dd}, J=9.2 \mathrm{~Hz}$ and $2.4 \mathrm{~Hz}), 8.77(1 \mathrm{H}, \mathrm{d}, J=2.4 \mathrm{~Hz}) .{ }^{13} \mathrm{C}-\mathrm{NMR}$ $\left(\mathrm{CDCl}_{3}\right) \delta 11.7\left(\mathrm{CH}_{3}\right), 22.7\left(\mathrm{CH}_{2}\right), 26.4\left(\mathrm{CH}_{2}\right), 27.3\left(\mathrm{CH}_{2}\right), 27.9\left(\mathrm{CH}_{2}\right), 30.0\left(\mathrm{CH}_{2}\right), 32.4\left(\mathrm{CH}_{2}\right), 43.7$ $\left(\mathrm{CH}_{2}\right), 112.4(\mathrm{C}), 118.4(\mathrm{CH}), 121.5(\mathrm{C}), 126.6(\mathrm{CH}), 130.2(\mathrm{CH}), 143.8(\mathrm{C}), 144.7(\mathrm{C}), 153.6(\mathrm{C})$, 160.8 (C), 164.3 (C). HR MS (+ESI): $m / z 361.1426\left(\mathrm{M}+\mathrm{H}^{+}\right)$. Calcd for $\mathrm{C}_{18} \mathrm{H}_{21} \mathrm{ClN}_{4} \mathrm{O}_{2}: 360.1353$.

(Z)-2-(1-Chloroprop-1-enyl)-6-nitro-N-propylquinazolin-4-amine (12): The eluent was dichloromethane. Green solid; yield 56\%, mp $176{ }^{\circ} \mathrm{C} .{ }^{1} \mathrm{H}-\mathrm{NMR}\left(\mathrm{CDCl}_{3}\right) \delta 1.03-1.10(3 \mathrm{H}, \mathrm{m}), 1.72-1.90(2 \mathrm{H}$, $\mathrm{m}), 2.08(3 \mathrm{H}, \mathrm{d}, J=7.0 \mathrm{~Hz}), 3.67-3.77(2 \mathrm{H}, \mathrm{m}), 6.43(1 \mathrm{H}$, br s), $7.63(1 \mathrm{H}, \mathrm{q}$, $J=7.0 \mathrm{~Hz}), 7.92-7.98(1 \mathrm{H}, \mathrm{m}), 8.44(1 \mathrm{H}, \mathrm{dd}, J=9.2 \mathrm{~Hz}$ and $2.4 \mathrm{~Hz})), 8.78(1 \mathrm{H}, \mathrm{d}, J=2.4 \mathrm{~Hz}) .{ }^{13} \mathrm{C}-$ NMR $\left(\mathrm{CDCl}_{3}\right) \delta 11.8\left(\mathrm{CH}_{3}\right), 15.8\left(\mathrm{CH}_{3}\right), 22.5\left(\mathrm{CH}_{2}\right), 43.9\left(\mathrm{CH}_{2}\right), 112.9(\mathrm{C}), 118.9(\mathrm{CH}), 126.9(\mathrm{CH})$, $129.4(\mathrm{CH}), 132.4(\mathrm{C}), 134.5(\mathrm{CH}), 144.6(\mathrm{C}), 152.5$ (C), $160.0(\mathrm{C}), 160.5(\mathrm{C})$. HR MS (+ESI): $m / z$ $307.0956\left(\mathrm{M}+\mathrm{H}^{+}\right)$. Calcd for $\mathrm{C}_{14} \mathrm{H}_{15} \mathrm{ClN}_{4} \mathrm{O}_{2}: 306.0884$.

(Z)-2-(1-Chlorobut-1-enyl)-6-nitro-N-propylquinazolin-4-amine (13): The eluent was dichloromethane. Yellow solid; yield 50\%, mp $163{ }^{\circ} \mathrm{C} .{ }^{1} \mathrm{H}-\mathrm{NMR}\left(\mathrm{CDCl}_{3}\right) \delta 0.98-1.23(6 \mathrm{H}, \mathrm{m}), 1.77-1.88(2 \mathrm{H}$, m), 2.46-2.61 (2H, m), 3.68-3.78 (2H, m), $6.40(1 \mathrm{H}$, br s), $7.55(1 \mathrm{H}, \mathrm{t}, J=9.3 \mathrm{~Hz}), 7.97(1 \mathrm{H}, \mathrm{d}$, $J=9.2 \mathrm{~Hz}), 8.45(1 \mathrm{H}, \mathrm{dd}, J=9.2 \mathrm{~Hz}$ and $2.4 \mathrm{~Hz}), 8.78(1 \mathrm{H}, \mathrm{d}, J=2.4 \mathrm{~Hz}) .{ }^{13} \mathrm{C}-\mathrm{NMR}$ $\left(\mathrm{CDCl}_{3}\right) \delta 11.2\left(\mathrm{CH}_{3}\right), 12.2\left(\mathrm{CH}_{3}\right), 22.0\left(\mathrm{CH}_{2}\right), 22.9\left(\mathrm{CH}_{2}\right), 43.1\left(\mathrm{CH}_{2}\right), 112.3(\mathrm{C}), 118.0(\mathrm{CH}), 126.1$ 
$(\mathrm{CH}), 129.3(\mathrm{CH}), 130.8(\mathrm{C}), 139.8(\mathrm{CH}), 143.8(\mathrm{C}), 152.6(\mathrm{C}), 159.4(\mathrm{C}), 160.3(\mathrm{C})$. Anal. Calcd for $\mathrm{C}_{15} \mathrm{H}_{17} \mathrm{ClN}_{4} \mathrm{O}_{2}$ : C, 56.16; H, 5.34; N, 17.47. Found: C, 55.82; H, 5.75; N, 17.01 .

(Z)-2-(1-Chloropent-1-enyl)-6-nitro-N-propylquinazolin-4-amine (14): $\quad$ The eluent was dichloromethane-ethyl acetate 99:1. Yellow solid; yield 52\%, mp $156{ }^{\circ} \mathrm{C} .{ }^{1} \mathrm{H}-\mathrm{NMR}$ (DMSO-d 6 ) $\delta$ 0.92-0.99 (6H, m), 1.49-1.76 (4H, m), 2.36-2.44 (2H, m), 3.50-3.60 (2H, m), 7.46-7.53 (1H, m), $7.81(1 \mathrm{H}, \mathrm{d}, J=9.1 \mathrm{~Hz}), 8.43(1 \mathrm{H}, \mathrm{dd}, J=9.1 \mathrm{~Hz}$ and $2.0 \mathrm{~Hz}), 9.01(1 \mathrm{H}, \mathrm{br}), 9.33(1 \mathrm{H}, \mathrm{d}$, $J=2.0 \mathrm{~Hz}) .{ }^{13} \mathrm{C}-\mathrm{NMR}\left(\mathrm{DMSO}_{-}\right) \delta 12.1\left(\mathrm{CH}_{3}\right), 14.2\left(\mathrm{CH}_{3}\right), 21.6\left(\mathrm{CH}_{2}\right), 21.9\left(\mathrm{CH}_{2}\right), 31.7\left(\mathrm{CH}_{2}\right), 43.2$ $\left(\mathrm{CH}_{2}\right), 113.5(\mathrm{C}), 120.9(\mathrm{CH}), 126.9(\mathrm{CH}), 129.7(\mathrm{CH}), 132.6(\mathrm{C}), 137.1(\mathrm{CH}), 144.3(\mathrm{C}), 153.8(\mathrm{C})$, 160.2 (C), 160.4 (C). HR MS (+ESI): $m / z 335.1269\left(\mathrm{M}+\mathrm{H}^{+}\right)$. Calcd for $\mathrm{C}_{16} \mathrm{H}_{19} \mathrm{ClN}_{4} \mathrm{O}_{2}: 334.1197$.

N-Butyl-2-(1-chloro-2-methylprop-1-enyl)-6-nitroquinazolin-4-amine (15): The eluent was petroleum ether-ethyl acetate 50:50. Beige solid; yield 57\%, mp $135{ }^{\circ} \mathrm{C} .{ }^{1} \mathrm{H}-\mathrm{NMR}\left(\mathrm{CDCl}_{3}\right) \delta 1.00(3 \mathrm{H}, \mathrm{t}$, $J=7.2 \mathrm{~Hz}), 1.40-1.58(2 \mathrm{H}, \mathrm{m}), 1.72-1.84(2 \mathrm{H}, \mathrm{m}), 2.05(3 \mathrm{H}, \mathrm{s}), 2.09(3 \mathrm{H}, \mathrm{s}), 3.75(2 \mathrm{H}, \mathrm{m}), 6.42(1 \mathrm{H}$, br s), $7.96(1 \mathrm{H}, \mathrm{d}, J=9.2 \mathrm{~Hz}), 8.49(1 \mathrm{H}, \mathrm{dd}, J=9.2$ and $2.3 \mathrm{~Hz}), 8.73(1 \mathrm{H}, \mathrm{d}, J=2.3 \mathrm{~Hz}) .{ }^{13} \mathrm{C}-\mathrm{NMR}$ $\left(\mathrm{CDCl}_{3}\right) \delta 13.8\left(\mathrm{CH}_{3}\right), 20.2\left(\mathrm{CH}_{2}\right), 22.2\left(\mathrm{CH}_{3}\right), 23.1\left(\mathrm{CH}_{3}\right), 31.3\left(\mathrm{CH}_{2}\right), 41.6\left(\mathrm{CH}_{2}\right), 112.3(\mathrm{C}), 118.2$ $(\mathrm{CH}), 124.4(\mathrm{C}), 126.4(\mathrm{CH}), 130.0(\mathrm{CH}), 137.4(\mathrm{C}), 144.5(\mathrm{C}), 153.3(\mathrm{C}), 160.5(\mathrm{C}), 164.0(\mathrm{C})$. Anal. Calcd for $\mathrm{C}_{16} \mathrm{H}_{19} \mathrm{ClN}_{4} \mathrm{O}_{2}$ : C, 57.40; H, 5.72; N, 16.73. Found: C, 57.02; H, 5.94; N, 16.43.

2-(1-Chloro-2-methylprop-1-enyl)-6-nitro-N-[3-(trifluoromethyl)]-phenylquinazolin-4-amine

(19):

The eluent was dichloromethane. Yellow solid, yield $45 \%, \mathrm{mp} 201{ }^{\circ} \mathrm{C} .{ }^{1} \mathrm{H}-\mathrm{NMR}\left(\mathrm{CDCl}_{3}\right) \delta 2.13(6 \mathrm{H}, \mathrm{s})$, 7.45-7.60 (2H, m), 7.98-8.11 (3H, m), $8.40(1 \mathrm{H}, \mathrm{br} \mathrm{s}), 8.58(1 \mathrm{H}, \mathrm{dd}, J=9.1 \mathrm{~Hz}$ and $2.2 \mathrm{~Hz}), 9.07(1 \mathrm{H}$, $\mathrm{d}, J=1.7 \mathrm{~Hz}) .{ }^{13} \mathrm{C}-\mathrm{NMR}\left(\mathrm{CDCl}_{3}\right) \delta 22.5\left(\mathrm{CH}_{3}\right), 23.7\left(\mathrm{CH}_{3}\right), 112.3(\mathrm{C}), 118.0(\mathrm{CH}), 118.8(\mathrm{CH}, \mathrm{q}$, $J=4.0 \mathrm{~Hz}), 121.6(\mathrm{CH}, \mathrm{q}, J=4.0 \mathrm{~Hz}), 123.8(\mathrm{C}, \mathrm{q}, J=272.6 \mathrm{~Hz}), 124.1(\mathrm{C}), 124.7(\mathrm{CH}), 127.0(\mathrm{CH})$, $129.7(\mathrm{CH}), 130.6(\mathrm{CH}), 131.6(\mathrm{C}, \mathrm{q}, J=32.6 \mathrm{~Hz}), 138.0(\mathrm{C}), 140.1(\mathrm{C}), 145.2(\mathrm{C}), 153.3(\mathrm{C}), 158.2$ (C), 163.1 (C). Anal. calcd for $\mathrm{C}_{19} \mathrm{H}_{14} \mathrm{ClF}_{3} \mathrm{~N}_{4} \mathrm{O}_{2}$ : C, 53.98; H, 3.34; N, 13.25. Found: C, 54.4; H, 3.52; N, 12.95 .

\subsection{Preparation of 6-nitro-2-trichloromethyl-N-[(3-trifluoromethyl)]phenylquinazolin-4-amine (18)}

Three equiv. of 3-trifluoromethylaniline were added onto 1 equiv. (500 mg) of 4-chloro-6-nitro-2trichloromethylquinazoline (17). The solvent-free reaction mixture was stirred at $120^{\circ} \mathrm{C}$ for $15 \mathrm{~min}$. The mixture was poured into a saturated solution of sodium carbonate and extracted with dichloromethane. The organic layer was washed with water three times, dried over anhydrous $\mathrm{Na}_{2} \mathrm{SO}_{4}$ and concentrated in vacuo. After purification by chromatography (silica gel, eluent: dichloromethane) the product was obtained in $74 \%$ yield. Yellow solid; yield $74 \%, \mathrm{mp} 234{ }^{\circ} \mathrm{C} .{ }^{1} \mathrm{H}-\mathrm{NMR}$ (DMSO-d 6 ) $\delta$ $7.56(1 \mathrm{H}, \mathrm{d}, J=7.7 \mathrm{~Hz}), 7.67-7.75(1 \mathrm{H}, \mathrm{m}), 8.14(1 \mathrm{H}, \mathrm{d}, J=9.2 \mathrm{~Hz}), 8.22(1 \mathrm{H}, \mathrm{d}, J=8.3 \mathrm{~Hz})$, 8.65-8.71 (2H, m), $9.80(1 \mathrm{H}, \mathrm{d}, J=2.4 \mathrm{~Hz}), 11.05(1 \mathrm{H}, \mathrm{br} \mathrm{s}) .{ }^{13} \mathrm{C}-\mathrm{NMR}\left(\mathrm{DMSO}-\mathrm{d}_{6}\right) \delta 97.2(\mathrm{C}), 113.7$ (C), $118.7(\mathrm{CH}, \mathrm{q}, J=4.0 \mathrm{~Hz}), 120.8(\mathrm{CH}), 120.9(\mathrm{CH}), 124.1\left(\mathrm{CF}_{3}, \mathrm{q}, J=272.7 \mathrm{~Hz}\right), 125.5(\mathrm{CH})$, $127.8(\mathrm{CH}), 129.3(\mathrm{C}, \mathrm{q}, J=31.9 \mathrm{~Hz}), 129.8(\mathrm{CH}), 130.4(\mathrm{CH}), 138.9(\mathrm{C}), 146.0(\mathrm{C}), 152.5(\mathrm{C}), 159.5$ (C), 162.7 (C). Anal. calcd for $\mathrm{C}_{16} \mathrm{H}_{8} \mathrm{Cl}_{3} \mathrm{~F}_{3} \mathrm{~N}_{4} \mathrm{O}_{2}$ : C, 42.55; H, 1.79; N, 12.41. Found: C, 42.50; H, $1.91 ; \mathrm{N}, 11.97$. 


\subsection{Preparation of 2-(1-chloro-2-methylprop-1-enyl)-6-nitroquinazolin-4(3H)-one (21)}

To a DMSO solution (15 mL) of the lithium salt of 2-nitropropane (0.76 g, $8.1 \mathrm{mmol}, 5$ equiv.), a DMSO solution $(15 \mathrm{~mL})$ containing $0.5 \mathrm{~g}$ (1.6 mmol, 1 equiv.) of 20 was added rapidly. The mixture was stirred at $\mathrm{rt}$ for $2 \mathrm{~h}$ under $\mathrm{N}_{2}$ atmosphere and light irradiation $(60 \mathrm{~W})$. After the disappearance of 20 (monitored by TLC), the mixture was poured into cold water, filtered, and extracted with dichloromethane. The combined organic layer were dried over anhydrous $\mathrm{Na}_{2} \mathrm{SO}_{4}$ and evaporated. The solid product was washed with petroleum ether and dried under reduced pressure to give 21 in $67 \%$ yield. Yellow solid; yield 67\%, mp $248{ }^{\circ} \mathrm{C} .{ }^{1} \mathrm{H}-\mathrm{NMR}\left(\mathrm{CDCl}_{3}\right) \delta 2.17(3 \mathrm{H}, \mathrm{s}), 2.30(3 \mathrm{H}, \mathrm{s}), 7.84(1 \mathrm{H}, \mathrm{d}$, $J=9.0 \mathrm{~Hz}), 8.56(1 \mathrm{H}, \mathrm{dd}, J=9.0 \mathrm{~Hz}$ and $2.4 \mathrm{~Hz}), 8.76(1 \mathrm{H}, \mathrm{d}, J=2.4 \mathrm{~Hz}), 9.74(1 \mathrm{H}, \mathrm{br} \mathrm{s}) .{ }^{13} \mathrm{C}-\mathrm{NMR}$ $\left(\mathrm{CDCl}_{3}\right) \delta 23.2\left(\mathrm{CH}_{3}\right), 24.3\left(\mathrm{CH}_{3}\right), 117.0(\mathrm{C}), 121.3(\mathrm{C}), 123.1(\mathrm{CH}), 128.9(\mathrm{CH}), 129.4(\mathrm{CH}), 146.2$ (C), $151.7(\mathrm{C}), 152.3(\mathrm{C}), 160.4(\mathrm{C}),\left(\mathrm{C}-\mathrm{NO}_{2}\right)$ was not observed in these experimental conditions. Anal. Calcd for $\mathrm{C}_{12} \mathrm{H}_{10} \mathrm{ClN}_{3} \mathrm{O}_{3}$ : C, 51.53; H, 3.60; N, 15.02. Found: C, 50.99; H, 3.53; N, 14.94 .

\section{Conclusions}

Quinazoline derivatives are molecules with a high pharmaceutical potential, particularly when targeting kinases. Using the consecutive $\mathrm{S}_{\mathrm{RN}} 1+\mathrm{E}_{\mathrm{RC}} 1$ radical reaction strategy, a series of original 4-propylaminoquinazolines, bearing a vinylic chloride group at position 2, was prepared in a simple and convenient way from corresponding trichloromethylated substrates. The reaction parameters were studied and optimized by using a phase-transfer procedure involving 5 equiv. of both TBAOH and 2-nitropropane, and the radical mechanism was investigated by employing radical reaction inhibitors such as TEMPO. Finally, the reaction was extended in 4-butylaminoquinazoline, 4-anilinoquinazoline and quinazoline- $4(3 H)$-one series, demonstrating that the $\mathrm{S}_{\mathrm{RN}} 1+\mathrm{E}_{\mathrm{RC}} 1$ reaction operated onto nitrated quinazolines is compatible with the presence of an alkylamino electrodonating group, so as with a lactam function.

\section{Acknowledgements}

This work was supported by the CNRS and the Université de la Méditerranée. The authors thank V. Remusat for recording NMR spectra.

\section{References}

1. Verhaeghe, P.; Azas, N.; Gasquet, M.; Hutter, S.; Ducros, C.; Laget, M.; Rault, S.; Rathelot, P.; Vanelle, P. Synthesis and antiplasmodial activity of new 4-aryl-2-trichloromethylquinazolines. Bioorg. Med. Chem. Lett. 2008, 18, 396-401.

2. Verhaeghe, P.; Azas, N.; Hutter, S.; Castera-Ducros, C.; Laget, M.; Dumètre, A.; Gasquet, M.; Reboul, J.P.; Rault, S.; Rathelot, P.; Vanelle, P. Synthesis and in vitro antiplasmodial evaluation of 4-anilino-2-trichloromethylquinazolines. Bioorg. Med. Chem. 2009, 17, 4313-4322.

3. Kabri, Y.; Azas, N.; Dumetre, A.; Hutter, S.; Laget, M.; Verhaeghe, P.; Gellis, A.; Vanelle, P. Original quinazoline derivatives displaying antiplasmodial properties. Eur. J. Med. Chem. 2010, $45,616-622$. 
4. Ryabukhin, S.V.; Plaskon, A.S.; Volochnyuk, D.M.; Tolmachev, A.A. Chlorotrimethylsilanemediated synthesis of 2-aryl-1-chloro-1-heteroarylalkenes. Synthesis 2007, 20, 3163-3170.

5. Vanelle, P.; Rathelot, P.; Maldonado, J.; Crozet, M.P. Consecutive $\mathrm{S}_{\mathrm{RN}} 1$ and $\mathrm{E}_{\mathrm{RC}} 1$ reactions in 5nitroisoquinolines. Tetrahedron Lett. 1994, 35, 8385-8388.

6. Vanelle, P.; Benakli, K.; Giraud, L.; Crozet, M.P. Synthesis of tetrasubstituted ethylenic compounds from a gem-trichloro imidazole derivative by electron-transfer reactions. Synlett 1999, 6, 801-803.

7. Verhaeghe, P.; Rathelot, P.; Rault, S.; Vanelle, P. Convenient preparation of original vinylic chlorides with antiparasitic potential in quinoline series. Lett. Org. Chem. 2006, 3, 891-897.

8. Kabri, Y.; Gellis, A.; Vanelle, P. Microwave-assisted synthesis in aqueous medium of new quinazoline derivatives as anticancer precursors. Green Chem. 2009, 11, 201-208.

9. Verhaeghe, P.; Rathelot, P.; Gellis, A.; Rault, S.; Vanelle, P. Highly efficient microwave assisted $\alpha$-trichlorination reaction of $\alpha$-methylated nitrogen containing heterocycles. Tetrahedron 2006, 62, 8173-8176.

10. Kerber, R.C.; Urry, G.W.; Kornblum, N. Radical anions as intermediates in substitution reactions. Carbon alkylation of nitroparaffin salts. J. Am. Chem. Soc. 1964, 86, 3904.

11. Burt, B.; Freeman, D.J.; Gray, P.G.; Norris, R.K.; Randles, D. Radical and ionic reactions of tetrabutylammonium aci-nitronates. Tetrahedron Lett. 1977, 35, 3063-3066.

12. Dehoff, L.H. Nitro- and chloro-derivatives of $\beta$-methyl- $\gamma$-oxyquinazoline (anhydroacetylorthamidobenzamide). J. Prakt. Chem. 1890, 42, 346-360.

13. Wan, Z.K.; Wacharasindhu, S.; Levins, C.G.; Lin, M.; Tabei, K.; Mansour, T.S. The scope and mechanism of phosphonium-mediated $\mathrm{S}_{\mathrm{N}} \mathrm{Ar}$ reactions in heterocyclic amides and ureas $J$. Org. Chem. 2007, 72, 10194-10210.

14. Girdler, D.J.; Norris, R.K. Unusual reaction of benzylidine diacetates. Ionic chain mechanism for the substitution of an acetate group by the 2-nitro-2-propanide ion. Tetrahedron lett. 1975, 7, 431-434.

15. Ono, N.; Miyake, H.; Tamura, R.; Hamamoto, I.; Kaji, A. Free radical chain elimination reaction $\left(E_{R C} 1\right)$. Conversion of vicinal dinitro compounds or $\beta$-nitro sulfones to olefins with tributyltin hybride. Chem. Lett. 1981, 10, 1139-1142.

16. Kornblum, N.; Boyd, S.D.; Pinnick, H.W.; Smith, R.G. New synthesis of olefins. J. Am. Chem. Soc. 1971, 93, 4316-4318.

17. Bogert, M.T.; Geiger, G.A. Researches on quinazolines (thirtieth paper). Study of the bromination and nitration of 4-quinazolines; The corresponding aminoquinazolines, and certain other new 4quinazolines. J. Am. Chem. Soc. 1912, 34, 524-534.

18. Hepworth, W. Quinazoline derivatives. Great Britain Patent 857362, 1960; [Chem. Abstr. 1961, $55,76248]$.

Sample Availability: Samples of the compounds are available from the authors.

(C) 2010 by the authors; licensee Molecular Diversity Preservation International, Basel, Switzerland. This article is an open-access article distributed under the terms and conditions of the Creative Commons Attribution license (http://creativecommons.org/licenses/by/3.0/). 